Tropical Journal of Pharmaceutical Research May 2017; 16 (5): 1039-1044

ISSN: $1596-5996$ (print); 1596-9827 (electronic)

(C) Pharmacotherapy Group, Faculty of Pharmacy, University of Benin, Benin City, 300001 Nigeria.

All rights reserved.

Available online at http://www.tjpr.org

Original Research Article

http://dx.doi.org/10.4314/tjpr.v16i5.10

\title{
Effect of a heme oxygenase-1 inducer on NADPH oxidase expression in alcohol-induced liver injury in male Wistar rats
}

\author{
Phanit Koomhin ${ }^{1,2}$, Chuchard Punsawad ${ }^{1}$, Prasit Suwannalert ${ }^{3}$ and Sarawoot \\ Palipoch ${ }^{1,2 *}$
}

${ }^{1}$ School of Medicine, ${ }^{2}$ The Pathobiology of Cell and Tissue Research Group, Walailak University, Nakhon Si Thammarat, $80161,{ }^{3}$ Department of Pathobiology, Faculty of Science, Mahidol University, Bangkok 10400, Thailand

*For correspondence: Email: spalipoch@hotmail.com, sarawoot.pa@wu.ac.th; Tel: +66 75672873

\begin{abstract}
Purpose: To investigated the effect of hemin, a heme oxygenase-1 (HO-1) inducer, on nicotinamide adenine dinucleotide phosphate oxidase (NOX) expression in rats with alcohol-induced liver injury.

Methods: Male Wistar rats were randomly divided into four groups consisting of the control group, the ethanol (EtOH) group, the EtOH + zinc protoporphyrin IX (ZnPP-IX) group and EtOH + hemin group. Hepatic NOX gene expression and immunohistochemistry of hepatic NOX1 and NOX4 were investigated in week 4.

Results: EtOH significantly increased levels of NOX. An immunohistochemical study demonstrated a high number of immunopositive hepatocytes for NOX1 in the EtOH group and EtOH + ZnPP-IX group compared with the control group. Hemin administration downregulated NOX gene expression and lowered the number of immunopositive hepatocytes for NOX1. In contrast, ZnPP-IX (HO-1 inhibitor) administration caused upregulation of NOX gene expression and increased the number of immunopositive hepatocytes for NOX1.

Conclusion: HO-1 inducer, hemin, alleviates oxidative stress-induced alcoholic liver injury by reducing NOX, especially NOX1.
\end{abstract}

Keywords: NADPH oxidase, Immunohistochemistry, Heme oxygenase-1, Hemin, Reactive oxygen species, Alcohol-induced liver disease

Tropical Journal of Pharmaceutical Research is indexed by Science Citation Index (SciSearch), Scopus, International Pharmaceutical Abstract, Chemical Abstracts, Embase, Index Copernicus, EBSCO, African Index Medicus, JournalSeek, Journal Citation Reports/Science Edition, Directory of Open Access Journals (DOAJ), African Journal Online, Bioline International, Open-J-Gate and Pharmacy Abstracts

\section{INTRODUCTION}

Alcohol-induced liver disease is an increasing global health problem. Acute alcohol consumption leads to fatty liver. Although fatty liver is a reversible injury, its progression can develop into more severe liver problems including steatohepatitis and cirrhosis [1]. Previous studies showed that oxidative stress is an important factor contributing to the development of alcohol-induced liver injury [2]. Ethanol consumption has been shown to increase the production of reactive oxygen species (ROS)/reactive nitrogen species (RNS), decrease cellular antioxidant levels, and eventually enhance oxidative stress in many tissues, especially the liver [3]. ROS/RNS play the critical roles in damage to several macromolecules especially lipids, proteins and DNA, eventually causing alcohol-induced liver disease [4].

ROS mainly comprise superoxide radical $\left(\mathrm{O}_{2}{ }^{\circ}\right)$, hydroxyl radical $\left({ }^{\circ} \mathrm{OH}\right)$, hydrogen peroxide 
$\left(\mathrm{H}_{2} \mathrm{O}_{2}\right)$. A variety of stimuli can produce $\mathrm{O}_{2}{ }^{\cdot}$ through nicotinamide adenine dinucleotide phosphate oxidase (NOX) [5]. NOX1 and NOX4 are expressed in liver; NOX1 mainly produces $\mathrm{O}_{2}^{-*}$ whereas NOX4 directly produces $\mathrm{H}_{2} \mathrm{O}_{2}$ [6]. NOX1 bind with their structural subunits to form active complexes associated with p22phox, p47phox, p67phox, and active Rac. NOX4 is a constitutively active membrane-bound isoform associated with p22phox only [7]. Oxidative stress can cause increased expression of heme oxygenase-1 (HO-1) levels [8]. Recently, Palipoch et al. demonstrated that $\mathrm{HO}-1$ inducer hemin can relieve alcohol-induced liver injury, especially hepatic steatosis [9]. However, the underlying mechanisms of hemin remain unclear. Accordingly, this study aims to investigate the effect of HO-1 inducer hemin on NADPH oxidase expression in alcohol-induced liver injury in male Wistar rats.

\section{EXPERIMENTAL}

\section{Animals}

Male Wistar rat strains $(\mathrm{n}=24)$ (Rattus norvegicus) 12 weeks of age were purchased from the Division of Animal House, Faculty of Science, Prince of Songkla University, Thailand. Animal procedures were performed with the approval of the Animal Ethics Committee, Walailak University (protocol no. 003/2014) and followed the Guide for the Care and Use of Laboratory Animals, National Research Council [10]. The animals were provided free access to standard commercial rat diet and distilled water before the experiment.

Rats were randomly divided into four groups $(\mathrm{n}=$ 6 per group): 1) rats gavaged with normal saline (control group); 2) rats gavaged with ethanol (30 $\% \mathrm{v} / \mathrm{v}$ in saline, $5 \mathrm{~g} / \mathrm{kg}$ ) once a day for 4 weeks (the EtOH group); 3) rats gavaged with ethanol and intraperitoneally (i.p.) injected with zinc protoporphyrin IX (ZnPP-IX) $(20 \mu \mathrm{mol} / \mathrm{kg})$, a HO1 inhibitor, every week for 4 weeks (the $\mathrm{EtOH}+$ ZnPP-IX group); and 4) rats gavaged with ethanol and i.p. injected with hemin $(30 \mu \mathrm{mol} / \mathrm{kg})$, a HO-1 inducer, every week for 4 weeks (the $\mathrm{EtOH}+$ hemin group). Alcohol feeding was performed according to the procedure of Nan et al. [11]. In weeks 4, the rats were euthanized with an anesthetizing thiopental sodium overdose $(100 \mathrm{mg} / \mathrm{kg} \mathrm{BW})$. The abdominal cavity was then opened, and the liver was collected.

\section{Hepatic NOX gene expression by RT-PCR}

Hepatic total RNA was extracted using an RNeasy Mini Kit (Qiagen, Germany). The content and purity of RNA were measured using a UV spectrophotometer. Reverse transcriptionpolymerase chain reaction (RT-PCR) was performed for amplification of the NOX gene. The thermal cycling conditions were set up with an initial denaturation step at $95{ }^{\circ} \mathrm{C}$ for $15 \mathrm{~min}$ and at $94{ }^{\circ} \mathrm{C}$ for $1 \mathrm{~min}$. The second step included an annealing of primer at $65^{\circ} \mathrm{C}$ for $1 \mathrm{~min}$ and the third step comprised an extension at $72{ }^{\circ} \mathrm{C}$ for 1 $\mathrm{min}$. The final step comprised an elongation at 72 ${ }^{\circ} \mathrm{C}$ for $10 \mathrm{~min}$. The NOX primers were $5^{\prime}$ GGAAATAGAAAGTT GACTGGCCC-3', forward, and 5'-GTATGAGTGCCATCCAGAGCAG-3', reverse [12], and the beta actin primers were $5^{\prime}$ TTCTTTGCAGCTCCTTCGTTGCCG-3', forward, and 5'-TGGATGGCT ACGTACATGG CTGGG3', reverse [13]. The DNA samples were loaded into $2 \%$ gel agarose. When stained with ethidium bromide, the gel was visualized with an UV transilluminator. The amount of PCR product was detected using the GeneTools software with image analysis (Syngene, Frederick, MD, USA).

\section{Immunohistochemistry of hepatic NOX1 and NOX4}

The liver sections were de-paraffinized and rehydrated. Heat-induced epitope retrieval was performed in sodium citrate buffer solution, $\mathrm{pH}$ 6.0 (Vector Laboratories, CA, USA), using a microwave. Endogenous peroxidase activity was quenched in $3 \%$ hydrogen peroxide in distilled water. Subsequently, the sections were incubated with the blocking buffer (normal goat serum) at room temperature for $30 \mathrm{~min}$ for blocking nonspecific binding site. After draining off the blocking buffer, the liver sections were incubated with optimal primary antibody containing rabbit anti-mouse NOX1 (Santa Cruz Biotechnology Inc., USA) and rabbit anti-mouse NOX4 (Santa Cruz Biotechnology Inc., USA) in a humidified chamber at $4{ }^{\circ} \mathrm{C}$ overnight.

After washing them three times with phosphatebuffered saline (PBS), the sections were incubated with appropriately diluted secondary antibody (Santa Cruz Biotechnology Inc., USA) in a humidified chamber at room temperature for 30 min. After washing with PBS, avidin-biotin complex (ABC) conjugated with horseradish peroxidase (HRP) (Vectastain ABC Kit, USA) was added to the sections. Subsequently, the sections were washed with PBS, and then the DAB Kit (Vector Laboratories, USA) was applied for $3 \mathrm{~min}$. After counterstaining with Mayer's hematoxylin (Merck, Darmstadt, Germany), the liver sections were dehydrated and mounted [14] and semi-quantitatively examined under a light microscope. All slides were randomly evaluated 
in 50 microscopic fields at high magnification by two independent observers (SP and CP).

\section{Data analysis}

Statistical analysis was performed using IBM SPSS statistics (version 23). All data are presented as mean \pm standard error of the mean (SEM). Comparison between groups were performed using one-way analysis of variance (ANOVA). Post hoc comparison was performed using least significant difference (LSD) test. Significance level was set at $p<0.05$.

\section{RESULTS}

\section{HO-1 inducer hemin reduced NOX mRNA levels in ethanol - treated rats}

The rats treated with ethanol (the EtOH group) and the rats treated with ethanol and injected with zinc protoporphyrin IX (the EtOH + ZnPP-IX group) had significantly enhanced $(p<0.05)$ NOX mRNA levels in the liver compared with rats in the control group, as shown in Figure 1. The rats treated with hemin had statistically significantly reduced $(p<0.001)$ NOX mRNA levels compared with rats in the EtOH group and the $\mathrm{EtOH}+\mathrm{ZnPP}-\mathrm{IX}$ group.

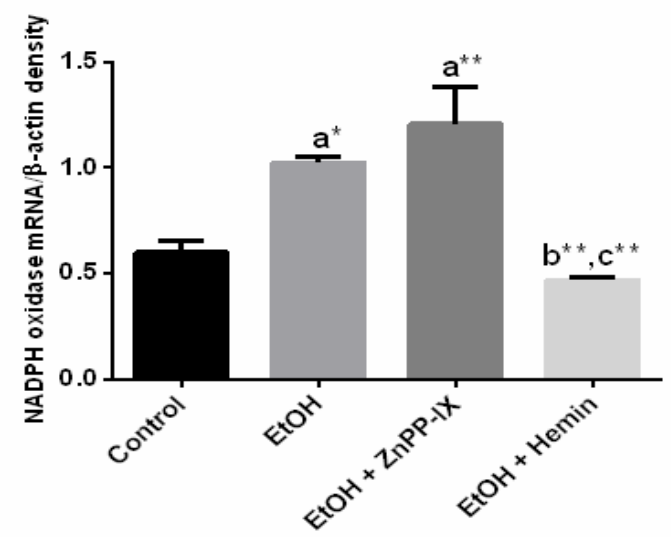

Figure 1: Hepatic NOX mRNA levels at week 4 (per group $n=6$ ). ${ }^{a, b, c}$ Compared with the control group, the $\mathrm{EtOH}$ group, and the $\mathrm{EtOH}+\mathrm{ZnPP}-\mathrm{IX}$ group, respectively. $p<0.05,{ }^{* *} p<0.001$

\section{HO-1 inducer hemin reduced hepatic NOX1}

Immunoperoxidase staining for NOX1 and NOX4 in rat hepatocytes at week 4 was illustrated in Figure 2 and Figure 3, respectively. The rats in the $\mathrm{EtOH}$ group and EtOH + ZnPP-IX group demonstrated a high number of immunopositive hepatocytes for NOX1 compared with the control group. The rats treated with $\mathrm{HO}-1$ inducer hemin demonstrated a lower number of immunopositive hepatocytes for NOX1 compared to rats in the
$\mathrm{EtOH}$ group and $\mathrm{EtOH}+\mathrm{ZnPP}-\mathrm{IX}$ group. In contrast, no difference in immunoreactivity for hepatic NOX4 was demonstrated among the groups.

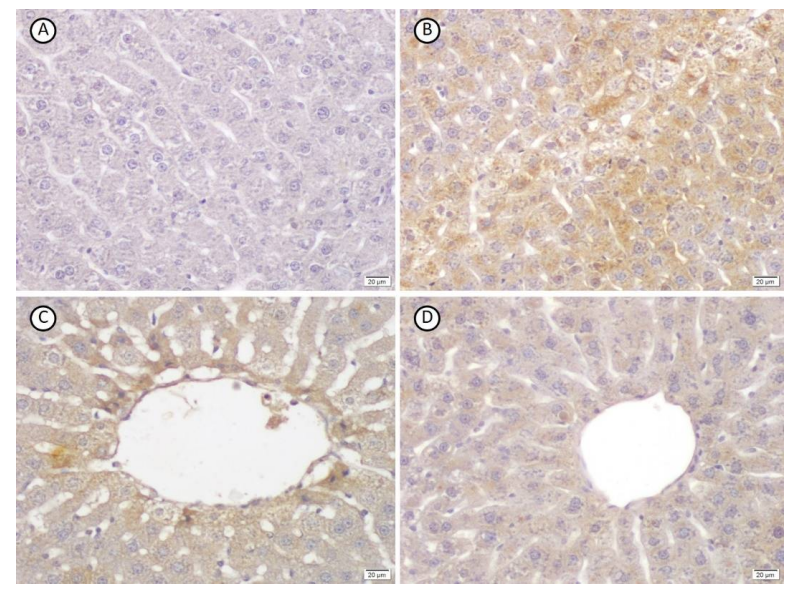

Figure 2: Immunoperoxidase staining for NOX1 in rat hepatocytes at week 4. (A) Rats in the control group. (B) Rats treated with ethanol. (C) Rats treated with an HO-1 inhibitor (ZnPP-IX) following ethanol gavage. (D) Rats treated with an $\mathrm{HO}-1$ inducer (hemin) following ethanol gavage. Scale bar $=20 \mu \mathrm{m}$

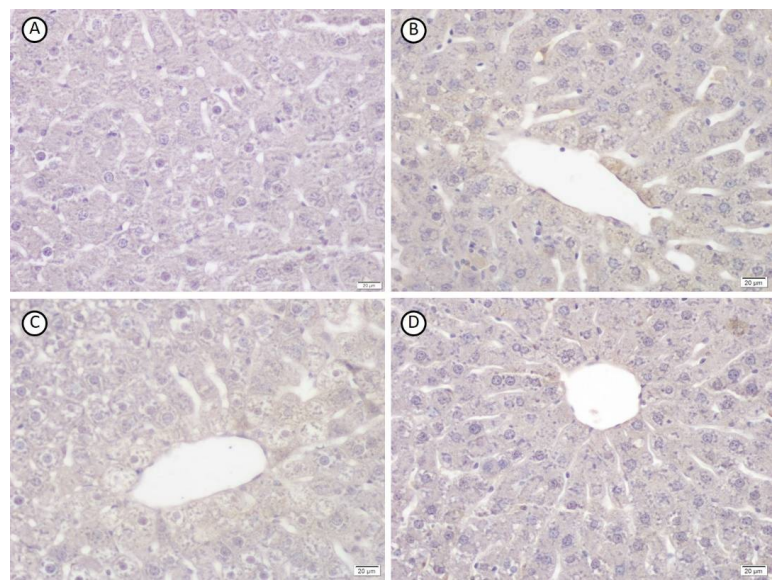

Figure 3: Immunoperoxidase staining for NOX4 in rat hepatocytes at week 4. (A) Rats in the control group. (B) Rats treated with ethanol. (C) Rats treated with an HO-1 inhibitor (ZnPP-IX) following ethanol gavage. (D) Rats treated with an HO-1 inducer (hemin) following ethanol gavage. Scale bar $=20 \mu \mathrm{m}$

As shown in Figure $4 \mathrm{~A}$, the percentage of positive stained hepatocytes for NOX1 was significant enhanced $(p<0.001)$ for the ethanoltreated rats compared with rats in the control group. The rats treated with an $\mathrm{HO}-1$ inhibitor (ZnPP-IX) following ethanol gavage had a significantly elevated $(p<0.001)$ percentage of positive stained hepatocytes compared with the ethanol-treated rats and rats in the control group. The rats treated with an $\mathrm{HO}-1$ inducer (hemin) following ethanol gavage had a significantly decreased percentage of positive stained 
hepatocytes compared with the ethanol-treated rats $(p<0.05)$ and HO-1 inhibitor-treated rats $(p$ $<0.001$ ). No difference in percentage of positive stained hepatocytes for NOX4 for male Wistar rats was demonstrated among the groups (Figure 4B).

A
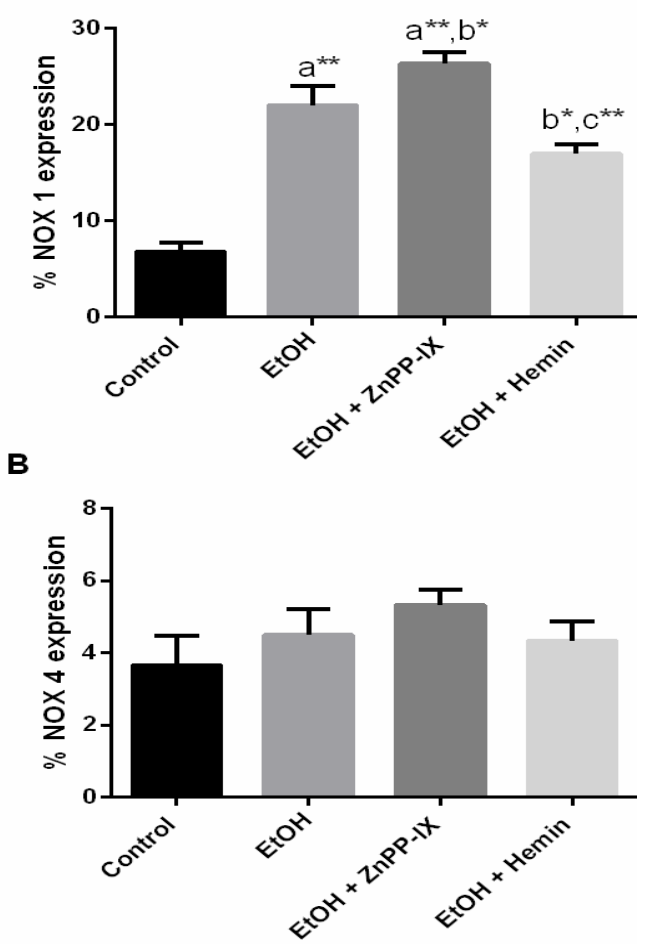

Figure 4: The percentage of positive stained hepatocytes for NOX1 (A) and NOX4 (B) at week 4 (per group $n=6$ ); ${ }^{a, b, c}$ compared with control group, the EtOH group and the EtOH + ZnPP-IX group, respectively. $p<0.05,{ }^{* *} p<0.001$

\section{DISCUSSION}

The liver is a major target organ of alcoholinduced injury. Oxidative stress is believed to involve liver damage induced by alcohol. Increased production of oxidants, including reactive oxygen species (ROS) and reactive nitrogen species (RNS) cause oxidative stress, which can damage cell components, including DNA, proteins and lipids [15]. Oxidation of ethanol leads to the pathology of liver disease, which comprises three major lesions and includes fatty liver, alcoholic hepatitis and cirrhosis, and eventually causes hepatocellular carcinoma. Research including in vitro, in vivo and clinical studies was investigated to understand the mechanism behind and develop a new approach to the management of alcoholinduced liver disease. Recently, Palipoch et al demonstrated that ethanol treatment of male Wistar rats by gavage for 4 weeks can induce fatty liver, with elevated levels of oxidative stress biomarker. This indicates oxidative stress is an important mechanism in inducing alcoholinduced liver injury [9].

Alcohol dehydrogenase (ADH) and cytochrome P450 2E1 (CYP2E1) are major pathways to oxidize ethanol to acetaldehyde. CYP2E1 is an effective generator of ROS [16]. Various endogenous sources such as inflammatory cells, respiratory chain, and nicotinamide adenine dinucleotide phosphate oxidase (NOX) can produce ROS [24]. Several types of NOX can be found in various cell types. NOX1 and NOX4 are the main ROS-producing NOX in hepatocytes [6]. A previous study demonstrated that CYP2E1 may play an important role in hepatocarcinogenesis induced by ethanol through causing oxidative stress to DNA, while NOX can produce oxidants involved in developing liver injury induced by ethanol [17]. The results of the present study indicate that NOX gene expression was elevated in the ethanol-treated rats, similar to previous studies $[18,19]$. Moreover, NOX1 was increased in liver sections of ethanol-treated rats detected by immunohistochemistry. We suggest that NOX1 is a key NOX that produces oxidants upon ethanol-induced liver injury.

Normally, oxidants are cleared via an antioxidant defense mechanism. Inefficiency of the antioxidant defense system causes oxidative stress-induced pathologies. Current studies have shown increased interest in investigating effective exogenous antioxidants to ameliorate oxidative stress-related pathologies. Heme oxygenase $(\mathrm{HO})$ is a critical stress-response protein that plays a key role in defense against oxidative stress [19-20]. Three different isoforms of mammalian $\mathrm{HO}$ have been reported, including inducible $\mathrm{HO}-1$, constitutive $\mathrm{HO}-2$, and $\mathrm{HO}-3$, which is not catalytically active [15]. HO-1 (heat shock protein 32 ) plays a potential role in cytoprotective stress and acts as a defensive mechanism against oxidative stress. HO-1 catalyzes the degradation of pro-oxidant heme to radical scavenging bile pigments, biliverdin, and bilirubin [22-24]. The present study demonstrates that an $\mathrm{HO}-1$ inducer inhibits NOX gene expression and reduces NOX1 expression in liver sections. We suggest that an HO-1 inducer alleviates oxidative stress-induced alcoholic liver injury through reducing NOX, especially, NOX1.

\section{CONCLUSION}

Oxidative stress is a major inducer of alcoholic liver injury through increased NOX gene expression and elevated expression of NOX1 in liver sections. An HO-1 inducer, hemin, alleviates 
oxidative stress-induced alcoholic liver injury by reducing NOX, especially NOX1. Thus, hemin may be useful in developing an effective antioxidant supplementation to ameliorate alcohol-induced liver injury.

\section{DECLARATIONS}

\section{Acknowledgement}

This research was supported by a grant from Institute of Research and Development (under contract WU57206), Walailak University, Thailand. We are thankful to the Dean of School of Medicine, Walailak University, for his kind support.

\section{Conflict of Interest}

No conflict of interest associated with this work.

\section{Contribution of Authors}

The authors declare that this work was done by the authors named in this article and all liabilities pertaining to claims relating to the content of this article will be borne by them.

\section{Open Access}

This is an Open Access article that uses a funding model which does not charge readers or their institutions for access and distributed under the terms of the Creative Commons Attribution License (http://creativecommons.org/licenses/by/ 4.0) and the Budapest Open Access Initiative (http://www.budapestopenaccessinitiative.org/rea d), which permit unrestricted use, distribution, and reproduction in any medium, provided the original work is properly credited.

\section{REFERENCES}

1. Louvet A, Mathurin P. Alcoholic liver disease: mechanisms of injury and targeted treatment. Nat Rev Gastroenterol Hepatol 2015; 12: 231-242.

2. Yang L, Rozenfeld $R$, Wu D, Devi LA, Zhang Z, Cederbaum A. Cannabidiol protects liver from binge alcohol-induced steatosis by mechanisms including inhibition of oxidative stress and increase in autophagy. Free Radic Biol Med 2014: 68; 260-267.

3. Dey A, Cederbaum Al. Alcohol and oxidative liver injury. Hepatology 200: 43; S63-74.

4. Muriel P. Role of free radicals in liver diseases. Hepatol Int 2009: 3; 526-536.

5. Palipoch $S$. A review of oxidative stress in acute kidney injury: protective role of medicinal plants-derived antioxidants. Afr J Tradit Complement Altern Med 2013: 10; 88-93.

6. Jiang JX, Török NJ. NADPH oxidases in chronic liver diseases. Adv Hepatol 2014: 2014; 742931.

7. Lambeth JD. NOX enzymes and the biology of reactive oxygen. Nat Rev Immunol 2004: 4; 181-189.

8. Le WD, Xie WJ, Appel SH. Protective role of heme oxygenase-1 in oxidative stress-induced neuronal injury. J Neurosci Res 1999: 56; 652-658.

9. Palipoch $S$, Koomhin $P$, Punsawad C, Na-Ek $P$, Sattayakhom A, Suwannalert $P$. Heme oxygenase-1 alleviates alcoholic liver steatosis: histopathological study. J Toxicol Pathol 2016: 29; 7-15.

10. Guide for the care and use of laboratory animals. National research council. 8th edition. Washington, DC: The national academies press, 2011.

11. Nan $Y$, Wang R, Zhao S, Han F, Wu WJ, Kong L, Fu N, $Y u$ J. Heme oxygenase-1 prevents non-alcoholic steatohepatitis through suppressing hepatocyte apoptosis in mice. Lipids Health Dis 2010: 9; 124.

12. Rashed LA, Hashem RM, Soliman HM. Oxytocin inhibits NADPH oxidase and P38 MAPK in cisplatin-induced nephrotoxicity. Biomed Pharmacother 2011: 65; 474480.

13. Bessa SS, Mohamed Ali EM, Abd El-Wahab Ael S, Nor El-Din SA. Heme oxygenase-1 mRNA expression in Egyptian patients with chronic liver disease. Hepat Mon 2012: 12; 278-285.

14. Punsawad C, Viriyavejakul $P$, Setthapramote $C$, Palipoch $S$. Enhanced expression of Fas and FasL modulates apoptosis in the lungs of severe $P$. falciparum malaria patients with pulmonary edema. Int J Clin Exp Pathol 2015: 8; 10002-10013.

15. Bhimaraj A, Tang WHW. Role of Oxidative Stress in Disease Progression in Stage B, a Pre-cursor of Heart Failure. Heart Failure Clinics 2012: 8; 101-111.

16. Lu Y, Cederbaum Al. CYP2E1 and oxidative liver injury by alcohol. Free Radic Biol Med 2008: 44; 723-738.

17. Bradford BU, Kono H, Isayama F, Kosyk O, Wheeler MD, Akiyama TE, Bleye L, Krausz KW, Gonzalez FJ, Koop DR, Rusyn I. Cytochrome P450 CYP2E1, but not nicotinamide adenine dinucleotide phosphate oxidase, is required for ethanol-induced oxidative DNA damage in rodent liver. Hepatology 2005: 41; 336-344.

18. De Minicis S, Brenner DA. Oxidative stress in alcoholic liver disease: role of NADPH oxidase complex. J Gastroenterol Hepatol 2008: Suppl 1; S98-103.

19. Kono $H$, Rusyn I, Yin M, Gäbele E, Yamashina S, Dikalova A, Kadiiska MB, Connor HD, Mason RP, Segal $B H$, Bradford BU, Holland SM, Thurman RG. NADPH oxidase-derived free radicals are key oxidants in alcohol-induced liver disease. J Clin Invest 2000: 106; 867-872.

20. Li L, Du G, Wang D, Zhou J, Jiang G, Jiang $H$. Overexpression of heme oxygenase-1 in mesenchymal stem Cells augments their protection on retinal cells in vitro and attenuates retinal ischemia/reperfusion injury in 
vivo against oxidative stress. Stem Cells Int 2017: 2017; 4985323.

21. Stocker $R$. Induction of haem oxygenase as a defence against oxidative stress. Free Radic Res Commun 1990: 9; 101-112.

22. Doré S, Takahashi M, Ferris CD, Zakhary R, Hester LD, Guastella $D$, Snyder SH. Bilirubin, formed by activation of heme oxygenase-2, protects neurons against oxidative stress injury. Proc Natl Acad Sci U S A 1999: 96; 2445-2450.
23. Nakagami T, Toyomura K, Kinoshita T, Morisawa S. A beneficial role of bile pigments as an endogenous tissue protector: anti-complement effects of biliverdin and conjugated bilirubin. Biochim Biophys Acta 1993: 1158; 189-193.

24. Stocker R, Yamamoto $Y$, McDonagh AF, Glazer AN, Ames BN. Bilirubin is an antioxidant of possible physiological importance. Science 1987: 235; 10431046. 